\title{
Combined analysis of the low-energy enhancement of the gamma-strength function and the giant dipole resonance
}

\author{
Cebo Ngwetsheni ${ }^{1}\left[\right.$. José Nicolás Orce ${ }^{1}$
}

Published online: 20 August 2019

(C) The Author(s) 2019

\begin{abstract}
The nuclear dipole polarizability is mainly governed by the dynamics of the giant dipole resonance and has been investigated along with the effects of the low-energy enhancement of the photon strength function for nuclides in medium- and heavy-mass nuclei. Cubic-spline interpolations to both data sets show a significant reduction of the nuclear dipole polarizability for semi-magic and doubly magic nuclei, with magic numbers $N=28,50,82$ and 126 , which supports shell effects at high-excitation energies from the quasi-continuum to the giant dipole resonance. This work expands on the data analysis of our recent publication in Ngwetsheni and Orce (Phys. Lett. B 792, 335, 2019), which reveals a new spectroscopic probe to search for "old" and "new" magic numbers at high-excitation energies. New results presented in this work suggest an even higher sensitivity of the nuclear polarizability to shell effects when extrapolating the low-energy enhancement at lower gamma-ray energies.
\end{abstract}

Keywords Nuclear dipole polarizability · Quasi-continuum · Low-energy enhancement · Photon-strength function · Photo-absorption cross sections · Shell model · Magic numbers

\section{Introduction}

Matter in the vicinity of an electromagnetic (EM) field tends to polarize as a result of a perturbation of the charge distribution. In the case of the nucleus, the polarizability is dominated

This article is part of the Topical Collection on Proceedings of the International Conference on Hyperfine Interactions and their Applications (HYPERFINE 2019), Goa, India, 10-15 February 2019 Edited by S. N. Mishra, P. L. Paulose and R. Palit

This work was supported by the National Research Foundation of South Africa, the MaNus/MatSci program and the SA-CERN collaboration.

Cebo Ngwetsheni

3344489@myuwc.ac.za

José Nicolás Orce

jnorce@uwc.ac.za

1 Department of Physics \& Astronomy, University of the Western Cape, P/B X17, Bellville, ZA-7535, South Africa 
by the dynamics of the isovector giant dipole resonance (GDR) [1, 2], which is observed as a wide peak - with a full width at half-maximum of about 4-5 MeV for closed-shell nuclei, which becomes broader as nuclei deform - in photo-absorption cross-section measurements. The GDR is a collective motion that can be initiated by reactions which favor $\Delta L=1$ and $\Delta T=1$, e.g. gamma absorption (real photons) or Coulomb excitation (virtual photons). The GDR is described macroscopically according to the liquid drop model as the inter-penetrating motion of proton and neutron fluids out of phase, resulting from the nuclear symmetry energy $a_{\text {sym }}$ in the Bethe-Weizsäcker semi-empirical mass formula [3, 4] acting as a restoring force [1],

$$
a_{\text {sym }}\left(\rho_{N}-\rho_{Z}\right)^{2} / \rho_{A},
$$

where $\rho_{N}, \rho_{Z}$ and $\rho_{A}$ are the neutron, proton and total mass densities, respectively. Using the liquid drop model, with potential energy $\rho_{Z}$, Migdal calculated the ground state (g.s.) electric dipole polarizability $\alpha_{E 1}=\frac{\mathbf{P}}{\mathbf{E}}$, where $\mathbf{P}$ is the electric dipole moment and $\mathbf{E}$ the electric field strength, connecting to $a_{\text {sym }}$ as follows,

$$
\alpha_{E 1}=\frac{e^{2} R^{2} A}{40 a_{\text {sym }}}=2.25 \times 10^{-3} A^{5 / 3} \mathrm{fm}^{3},
$$

where $a_{\text {sym }}=23 \mathrm{MeV}$ was assumed by Migdal as well as a defined spherical surface of radius $R=1.2 A^{1 / 3} \mathrm{fm}$ [1]. Hence, $\alpha_{E 1}$ is proportional to the size and diffuseness of the nucleus. As a second-order effect in perturbation theory, $\alpha_{E 1}$ is also related to the total photo-absorption cross section $\sigma_{\text {total }}$ and its (-2) moment, $\sigma_{-2}$, in the following manner [5],

$$
\alpha_{E 1}=2 e^{2} \sum_{n} \frac{\langle i\|\hat{E} 1\| n\rangle\langle n\|\hat{E} 1\| i\rangle}{E_{\gamma}}=\frac{\hbar c}{2 \pi^{2}} \sigma_{-2},
$$

where $\hat{E} 1$ is the electric dipole operator, $|i\rangle$ and $|n\rangle$ are the ground and excited state vectors and $\sigma_{-2}$ is defined as,

$$
\sigma_{-2}:=\int_{0}^{E_{\gamma_{\max }}} \frac{\sigma_{\text {total }}\left(E_{\gamma}\right)}{E_{\gamma}^{2}} d E_{\gamma},
$$

where $E_{\gamma_{\max }}$ is dependent on experiment (e.g., photo-neutron cross sections are measured above neutron threshold [6]). Additionally, a new empirical formula for $\sigma_{-2}$ [7] has been determined from the 1988 photoneutron cross-section evaluation using monoenergetic photons [6],

$$
\sigma_{-2}=2.4 \kappa A^{5 / 3} \mu \mathrm{b} / \mathrm{MeV},
$$

where the polarizability parameter $\kappa$ is included to account for deviations from the actual GDR effects to that predicted by the hydrodynamic model [1, 8]. The polarizability parameter $\kappa$ can therefore be extracted for known $\sigma_{-2}$ values and vice versa.

The sum rule in Eq. 3 indicates that large $E 1$ matrix elements via virtual excitations of the GDR [9] may polarize the shape of the ground state $|i\rangle$. Similarly, two-step processes of the type $|i\rangle \rightarrow|n\rangle \rightarrow|f\rangle$ (e.g. $0_{1}^{+} \rightarrow 1_{G D R}^{-} \rightarrow 2_{1}^{+}$) can polarize the shape of final excited states $|f\rangle$. This polarization phenomenon is the so-called E1 polarizability which is directly related to $\alpha$ - and may compete with the reorientation effect, RE; both being second-order effects in Coulomb-excitation theory [10-13]. The RE generates a timedependent hyperfine splitting of nuclear levels which depend on their shape, and can be used to determine spectroscopic quadrupole moments or $Q_{S}$ values [12] - i.e. the nuclear charge distribution in the laboratory frame - of states with angular momentum $J \neq 0, \frac{1}{2}[11,12]$. In fact, the $E 1$ polarizability gives rise to extra deformation, which may affect extracted 
reduced transition probabilities, i.e. $B(E 2)$ values, and shift $Q_{S}$ values toward more prolate shapes [14]. The determination of the polarizability parameter $\kappa$ is therefore relevant to the determination of collective properties such as $\mathrm{B}(\mathrm{E} 2)$ and $Q_{S}$ values $[15,16]$.

More generally, $\sigma_{-2}$ values should include both electric $\left(\alpha_{E 1}\right)$ and magnetic $\left(\chi_{M 1}\right)$ dipole polarizabilities [17],

$$
\sigma_{-2}=\frac{2 \pi^{2}}{\hbar c}\left(\alpha_{E 1}+\chi_{M 1}\right)
$$

Similarly, $\chi_{M 1}$ is a measure of the magnetic dipole response, $\mathbf{M}$, to a magnetic field, $\mathbf{B}$, i.e. $\mathbf{M}=\chi_{M 1} \cdot \mathbf{B}$ [18], and can be decomposed into paramagnetic $\left(\chi_{M 1}^{\text {para }}\right)$ and diamagnetic $\left(\chi_{M 1}^{\text {dia }}\right)$ susceptibilities,

$$
\chi_{M 1}=\chi_{M 1}^{p a r a}+\chi_{M 1}^{d i a}=2 \sum_{n} \frac{\langle i\|\hat{M} 1\| n\rangle\langle n\|\hat{M} 1\| i\rangle}{E_{\gamma}}-\frac{Z e^{2}}{6 m c^{2}}\left\langle r^{2}\right\rangle .
$$

Permanent magnetic dipole moments, i.e. paramagnetism, is dominant for $A<20$ nuclides and may have a substantial contribution to $\sigma_{-2}$ values (e.g. ${ }^{6} \mathrm{Li}$ and ${ }^{7} \mathrm{Li}$ ), whereas diamagnetism has negligible effects $[17,18]$.

Because of the $1 / E_{\gamma}^{2}$ weighting in Eq. $4, \sigma_{-2}$ values are sensitite to low-energy $E_{\gamma}$ and not significantly affected by nucleon resonances at high $E_{\gamma}$ (e.g. pion exchanges at $E_{\gamma} \gtrsim 140 \mathrm{MeV}$ ) [19]; hence, they are extremely sensitive measures - unlike $\sigma_{\text {total }}$ - of long-range correlations in the nuclear wave functions.

\section{Low energy enhancement of the photon strength function}

A potentially larger effect to $\sigma_{-2}$ values at higher excitation energies in the quasi-continuum region may arise from the low-energy enhancement (LEE) of the radiative or photon strength function $f\left(E_{\gamma}\right)$. The photon strength function $f\left(E_{\gamma}\right)$ characterizes average EM decay and absorption properties of excited nuclei. Recent measurements of $f\left(E_{\gamma}\right)$ by the Oslo group have revealed an enhancement at low $E_{\gamma}$ [20-23]. These measurements are performed in the quasi-continuum energy region and assumes the validity of the Brink-Axel hypothesis [24, 25], which states that $f\left(E_{\gamma}\right)$ is independent of the particular structure and only depends on $\mathrm{E}_{\gamma}$, i.e. GDR properties are similar for all initial nuclear states. To date, the EM character of the LEE remains undetermined experimentally, although polarization asymmetry measurements of $\gamma$ rays in ${ }^{56} \mathrm{Fe}$ show a dominant dipole radiation at $E_{\gamma}<1.5 \mathrm{MeV}$ [26]. Various interpretations of the LEE have been proposed, explaining its dipole origin as $M 1$ [27-32] and $E 1$ [33] dipole radiation [34]. Shell-model (SM) calculations consistently support the $M 1$ nature of the LEE [27-30]. The main purpose of this work is to quantify the potentially large contribution from the LEE anomaly to the nuclear polarizability and $\sigma_{-2}$ values assuming dipole radiation and validity of the Brink-Axel hypothesis.

\section{Systematics and results}

The LEE is generally observed in medium-mass nuclei in the $A \approx 50$ and 90 mass regions and only for ${ }^{105} \mathrm{Cd},{ }^{138,139} \mathrm{La}$ and ${ }^{151,153} \mathrm{Sm}$ [35-37] in heavy-mass nuclei. These nuclei, spanning the mass range $A=45-153$, have been considered in order to obtain a systematic 
study of LEE + GDR effects on $\sigma_{-2}$ values, which requires the combined analysis of LEE and GDR cross sections,

$$
\sigma_{\text {total }}\left(E_{\gamma}\right)=\sigma_{G D R}\left(E_{\gamma}\right)+\sigma_{L E E}\left(E_{\gamma}\right) \text {, }
$$

where $\sigma_{G D R}\left(E_{\gamma}\right)=\sigma(\gamma, p)+\sigma(\gamma, n)+\sigma(\gamma, 2 n)+\sigma(\gamma, n p)$ is given by photo-absorption reactions at energies above nucleon threshold, and $\sigma_{L E E}\left(E_{\gamma}\right)$ is the cross section contribution from the LEE region below the neutron threshold. The GDR data are obtained from the experimental nuclear-reaction databases EXFOR https://www-nds.iaea.org/exfor/exfor. htm and ENDF https://www.nndc.bnl.gov/ensdf/, whereas the LEE data come from the Oslo compilation of $f\left(E_{\gamma}\right)$ https://www.mn.uio.no/fysikk/english/research/about/infrastructure/ ocl/nuclear-physics-research/compilation/, in units of $\mathrm{MeV}^{-3}$. The LEE data can be converted to cross sections as follows [38],

$$
\sigma_{L E E}\left(E_{\gamma}\right)=\pi^{2} g_{J}(\hbar c)^{2} f\left(E_{\gamma}\right) E_{\gamma}[\mathrm{mb}],
$$

where $g_{J}$ is the statistical factor $g_{J}=\frac{2 J_{f}+1}{2 J_{i}+1}$ with spins $J_{i}$ and $J_{f}$ corresponding to initial and final states, respectively. The magnitude of $g_{J}$ affects $\sigma_{-2}$ and polarizability values proportionally. Considering the dipole character of the LEE, $g_{J}=1$ is a reasonable approximation for dipole transitions, particularly for $\Delta J=0$ and $\Delta J=1$ transitions. A value of $g_{J}=3$ is more suitable for even-even nuclei, resulting from $1^{-} \rightarrow 0^{+}$transitions in the GDR.

The combination of GDR and LEE contributions may be arguable, because $\sigma_{G D R}\left(E_{\gamma}\right)$ corresponds to transitions between excited states $|n\rangle$ in the GDR region and the g.s. $|i\rangle$, whereas $\sigma_{L E E}\left(E_{\gamma}\right)$ results from transitions between excited states in the quasi-continuum region. Recent studies of $f\left(E_{\gamma}\right)$ by Guttormsen and co-workers [39] in the LEE region support the validity of the Brink-Axel hypothesis at different excitation energies. This, together with the fact that GDR studies of hot nuclei (at relatively low temperatures $T$ and $\operatorname{spin} J$ ) and cold nuclei ( $T=0$ for the ground state) present similar features [9, 40], may allow for combining the LEE and GDR cross sections [41].

An interpolation method for calculating $\sigma_{\text {total }}\left(E_{\gamma}\right)$ and $\sigma_{-2}$ values has been used in this work. This method is independent of any physical phenomena and operates by creating a function - cubic or $4^{\text {th }}$ order polynomial - that interpolates between the fixed experimental data points. As an example, Fig. 1 shows the total cross section of ${ }^{56} \mathrm{Fe}$ with a cubic interpolation function (solid blue line). The resulting function is integrated accordingly to obtain $\sigma_{\text {total }}\left(E_{\gamma}\right)$, which yields the $\sigma_{-2}$ values listed in Table 1 and shown in Fig. 2.

Most nuclei present an energy gap (missing experimental data) between the LEE and GDR data, which may include the $M 1$ spin-flip resonance and the pygmy dipole resonances (PDR) for neutron-rich nuclei. Therefore, data from ENDF https://www.nndc.bnl. gov/ensdf/ - when available - have been used to fill the gap, as shown in Fig. 3a. Additionally, data near nucleon threshold energies generally present large uncertainties and have been excluded.

Because of minimal RMS errors, a cubic-spline interpolation has been selected as the interpolating function throughout this work. Similar results are obtained using a $4^{\text {th }}$-order polynomial interpolation. Lower and higher order interpolations present unexpected structures (bumps) in the energy-gap region and above. This is shown in Fig. $3 \mathrm{~b}$ for the ${ }^{45} \mathrm{Sc}$ data fitted to a quadratic interpolation function. The errors associated to $\sigma_{-2}$ values are calculated from upper and lower loci limits of $\sigma_{\text {total }}\left(E_{\gamma}\right)$, including LEE and GDR contributions as shown in Fig. 4, which yields an uncertainty of $7 \%$. Uncertainties for the interpolation data spanning the gap and extrapolated data are treated as three points standard deviation of the mean, in order to determine the upper and lower limits for these data sets. Most of the considered nuclei are stable, except ${ }^{50} \mathrm{~V},{ }^{138} \mathrm{La}$ and ${ }^{153} \mathrm{Sm}$ with no experimental GDR cross 


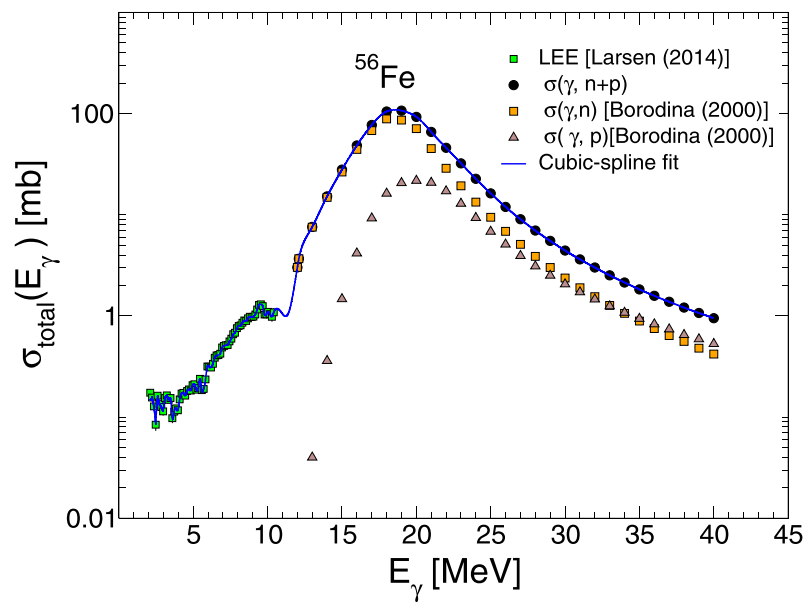

Fig. $1 \sigma_{\text {total }}\left(E_{\gamma}\right)$ vs $E_{\gamma}$ on a log scale on the y-axis showing the interpolation function data (solid blue line) for ${ }^{56} \mathrm{Fe}$, the green squares represent $\sigma_{L E E}\left(E_{\gamma}\right)$ and black filled circles the sum of proton and neutron emission cross sections for the GDR

Table 1 Contributions of GDR and LEE cross-sections to $\sigma_{-2}$ and $\kappa$ values

\begin{tabular}{|c|c|c|c|c|c|c|c|}
\hline Nucleus & $\begin{array}{l}\mathrm{E}_{\gamma(\max )}(\mathrm{GDR}) \\
(\mathrm{MeV})\end{array}$ & $\begin{array}{l}\mathrm{E}_{\gamma(\max )}(\mathrm{LEE}) \\
(\mathrm{MeV})\end{array}$ & $\begin{array}{l}\sigma_{-2}(\text { total }) \\
(\mu \mathrm{b} / \mathrm{MeV})\end{array}$ & $\begin{array}{l}\sigma_{-2}(\mathrm{LEE}) \\
(\mu \mathrm{b} / \mathrm{MeV})\end{array}$ & $\begin{array}{l}\text { C } \\
(\%)\end{array}$ & $\begin{array}{l}\kappa \\
\text { (with LEE) }\end{array}$ & [Refs.] \\
\hline${ }_{21}^{45} \mathrm{Sc}^{*}$ & 28.1 & 3.2 & $1840(130)$ & 178 & $9.7 \%$ & $1.35(9)$ & [42-44] \\
\hline${ }_{23}{ }^{1} \mathrm{~V}$ & 27.8 & 3.1 & $1458(100)$ & 43 & $2.9 \%$ & $0.89(5)$ & {$[45,46]$} \\
\hline${ }_{23} \mathrm{~V}$ & 27.8 & 3.1 & $1472(100)$ & 49 & $3.3 \%$ & $0.87(5)$ & {$[45,46]$} \\
\hline${ }_{26}^{56} \mathrm{Fe}^{*}$ & 40.0 & 3.8 & $2231(155)$ & 141 & $6.3 \%$ & $1.13(6)$ & {$[47,48]$} \\
\hline${ }_{32}^{76} \mathrm{Ge}$ & 26.5 & 2.3 & $3189(225)$ & 86 & $2.7 \%$ & $0.97(5)$ & {$[49,50]$} \\
\hline${ }_{40}^{92} \mathrm{Zr}$ & 27.8 & 2.2 & $3131(220)$ & 34 & $1.1 \%$ & $0.70(3)$ & [51-53] \\
\hline${ }_{42}^{95} \mathrm{Mo}$ & 27.8 & 2.5 & $4743(330)$ & 81 & $1.7 \%$ & $1.00(5)$ & {$[54,55]$} \\
\hline${ }_{57}^{138} \mathrm{La}$ & 24.3 & 1.9 & $7983(560)$ & 319 & $0.4 \%$ & $0.90(5)$ & {$[36,56]$} \\
\hline${ }_{57}^{139} \mathrm{La}$ & 24.3 & 2.5 & $8015(560)$ & 561 & $0.7 \%$ & $0.90(4)$ & {$[36,56]$} \\
\hline${ }_{62}^{153} \mathrm{Sm}$ & 20.0 & 1.6 & $9999(700)$ & 267 & $2.7 \%$ & $0.95(5)$ & {$[37,57]$} \\
\hline
\end{tabular}

Data have been extracted from EXFOR https://www-nds.iaea.org/exfor/exfor.htm, ENDF https:// www.nndc.bnl.gov/ensdf/ and the Oslo compilation http://www.mn.uio.no/fysikk/english/research/ $\backslash$ about/ infrastructure/OCL/nuclear-physics-research/compilation/. An asterisk indicates that the $\sigma_{-2}$ value includes $\sigma(\gamma, p)$ contributions. $\mathbf{C}$ is a measure of the LEE contribution to total $\sigma_{-2}$ values. This table is adapted from Ref. [41]

sections. Therefore, GDR data of stable neighboring isotopes were used, i.e. ${ }^{51} \mathrm{~V},{ }^{139} \mathrm{La}$ and ${ }^{152} \mathrm{Sm}$, under the assumption that neighboring nuclei present similar $f\left(E_{\gamma}\right)[22]$ and the fact that $\sigma_{-2}$ values show a strong dependence on nuclear mass $A$.

Setting up the low-energy cut-off, $E_{\gamma_{\min }}$, for the LEE is not obvious. In our previous study, we extrapolated the LEE data down to $800 \mathrm{keV}$ from experimental observations which show $E_{\gamma_{\min }} \approx 1 \mathrm{MeV}$ for most nuclei, except for ${ }^{153} \mathrm{Sm}$ where measurements were carried out down to $E_{\gamma_{\min }}=645 \mathrm{keV}$. The $\sigma_{-2}(\mathrm{LEE})$ values in Table 1 are calculated between the 

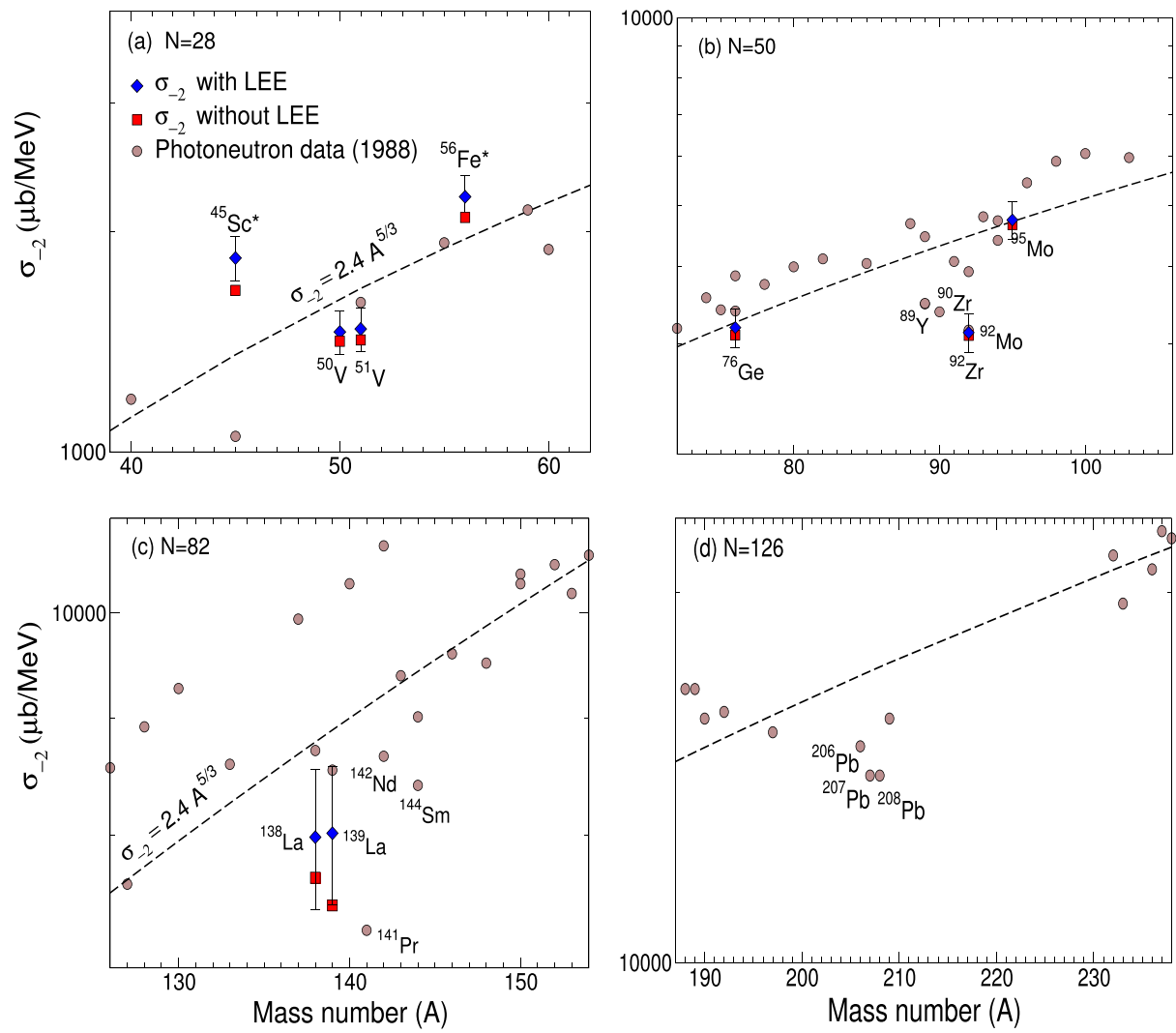

Fig. $2 \sigma_{-2}$ vs A on a $\log$ scale on the y-axis for nuclei around $\mathbf{a} N=28, \mathbf{b} N=50, \mathbf{c} N=82$ and $\mathbf{d}$ $N=126$. Data are from the photo-neutron cross-section evaluation (solid circles) [6] and $\sigma_{-2}$ values listed in Table 1, excluding (squares) and including (diamonds) the LEE contributions. For comparison, Eq. 5 (dashed line) is plotted
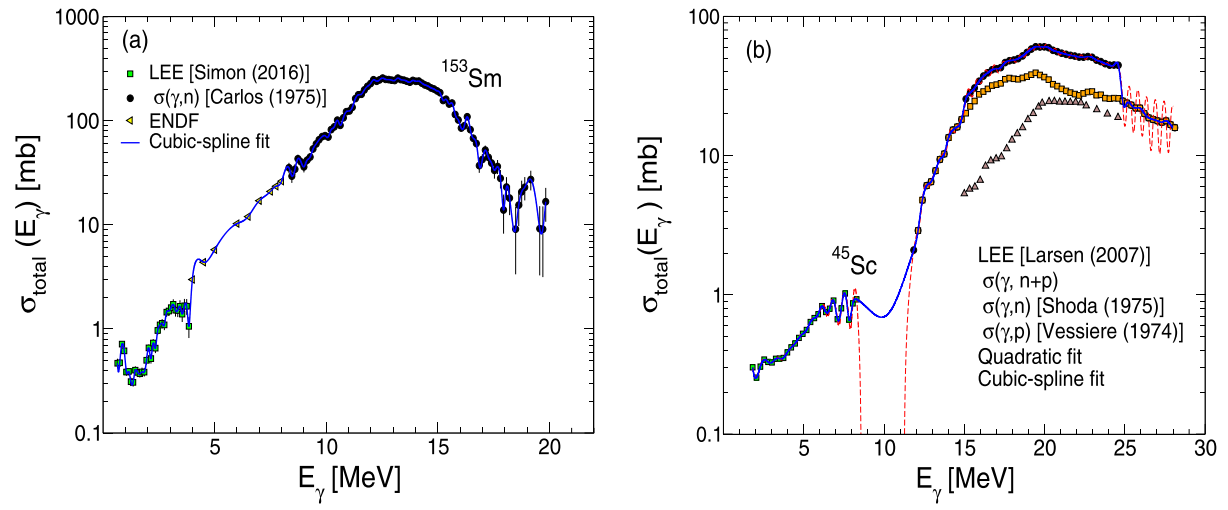

Fig. $3 \sigma_{\text {total }}\left(E_{\gamma}\right)$ vs $E_{\gamma}$ in log scale on the y-axis for a ${ }^{153} \mathrm{Sm}$ from ENDF data (triangles) and $\mathbf{b}{ }^{45} \mathrm{Sc}$ interpolated with a cubic (solid blue line) and quadratic (red dashed line) functions 


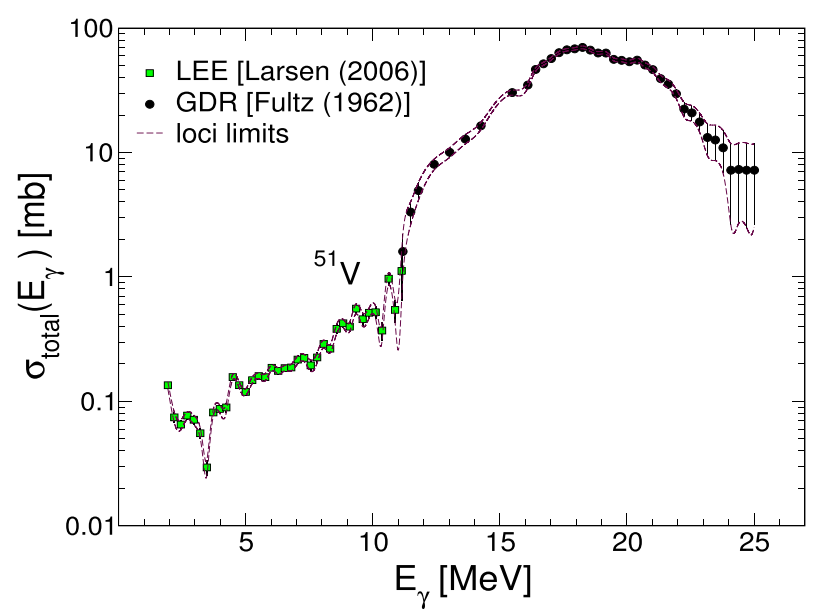

Fig. $4 \sigma_{\text {total }}\left(E_{\gamma}\right)$ vs $E_{\gamma}$ in $\log$ scale on the y-axis for ${ }^{51} \mathrm{~V}$ showing the upper and lower loci limits used to estimate the error

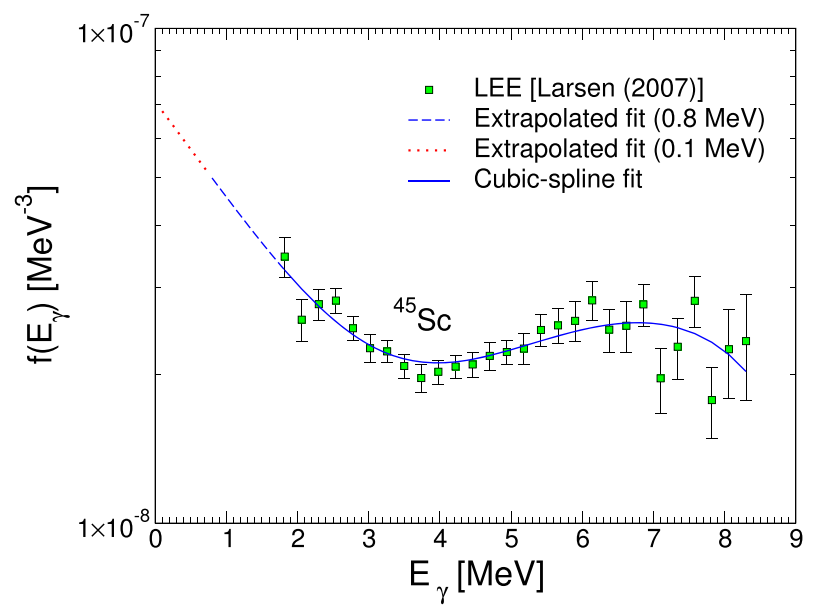

Fig. $5 f\left(E_{\gamma}\right)$ for ${ }^{45} \mathrm{Sc}$ fitted with a cubic polynomial (solid blue line), including extrapolated data to $E_{\gamma_{\min }}=$ $0.8 \mathrm{MeV}$ (dashed blue line) and $E_{\gamma_{\min }}=0.1 \mathrm{MeV}$ (red dotted line)

lower $E_{\gamma_{\min }}=0.8 \mathrm{MeV}$ and $E_{\gamma_{\max }}(\mathrm{LEE})$, where the LEE starts [41]. Recent SM studies [26, $29,31]$ explore, however, the behavior of $f\left(E_{\gamma}\right)$ at very low $E_{\gamma}$, supporting the continuation of the LEE down to $E_{\gamma_{\min }}=0$. Consequently, we have investigated this situation and Fig. 5 shows an extrapolated fit of $f\left(E_{\gamma}\right)$ to ${ }^{45} \mathrm{Sc}$ data down to $E_{\gamma_{\min }}=0.1 \mathrm{MeV}$. Similar fits were done for ${ }^{51} \mathrm{~V}$ and ${ }^{56} \mathrm{Fe}$ to explore $f\left(E_{\gamma}\right)$ in the $A \approx 50$ region and the results are listed in Table 2. A large enhancement of $\sigma_{-2}$ values is found for ${ }^{45} \mathrm{Sc}$ and ${ }^{56} \mathrm{Fe}$ as compared with ${ }^{51} \mathrm{~V}$. If these predictions of $f\left(E_{\gamma}\right)$ for $E_{\gamma_{\min }} \rightarrow 0 \mathrm{MeV}$ are consistent with experimental findings, reaction rates in nucleosynthesis following rapid-neutron capture - the $r$-process - may strongly be affected together with the predicted abundances of nuclei [36, 58-60]. 
Table 2 Contributions of the LEE with $E_{\gamma_{\min }}=0.1 \mathrm{MeV}$ represented by $\mathbf{C}$

\begin{tabular}{lllllll}
\hline Nucleus & $\begin{array}{l}\mathrm{E}_{\gamma(\text { min })} \\
(\mathrm{MeV})\end{array}$ & $\begin{array}{l}\mathrm{E}_{\gamma(\max )}(\mathrm{GDR}) \\
(\mathrm{MeV})\end{array}$ & $\begin{array}{l}\mathrm{E}_{\gamma(\max )}(\mathrm{LEE}) \\
(\mathrm{MeV})\end{array}$ & $\begin{array}{l}\sigma_{-2}(\text { total }) \\
(\mu \mathrm{b} / \mathrm{MeV})\end{array}$ & $\begin{array}{l}\sigma_{-2}(\mathrm{LEE}) \\
(\mu \mathrm{b} / \mathrm{MeV})\end{array}$ & $\begin{array}{l}\mathrm{C} \\
\%\end{array}$ \\
\hline${ }_{21}^{4} \mathrm{Sc}$ & 0.1 & 28 & 3.2 & 2488 & 873 & $35.1 \%$ \\
${ }_{51}^{1} \mathrm{~V}$ & 0.1 & 27.8 & 3.1 & 1564 & 140 & $9 \%$ \\
${ }_{56}^{56} \mathrm{Fe}$ & 0.1 & 40 & 3.8 & 2658 & 568 & $21.4 \%$ \\
\hline
\end{tabular}

$\mathrm{E}_{\gamma(\max )}(\mathrm{LEE})$ is the energy at which the LEE starts

\section{Discussion and conclusion}

As shown in our previous work [41], drops of nuclear polarizability are evident in nuclei with or near magic numbers $N=28,50,82$ and 126, which are characterized by values of $\kappa<1$. The work presented here expands on the data analysis presented in Ref. [41], but also provides an additional piece of evidence for shell effects. As illustrated when comparing Tables 1 and 2 for the neighboring ${ }^{45} \mathrm{Sc},{ }^{51} \mathrm{~V}$ and ${ }^{56} \mathrm{Fe}$ nuclei, it is clear that once we extrapolate the LEE down to $E_{\gamma}=100 \mathrm{keV}$ (see Table 2), $\sigma_{-2}$ values show a higher sensitivity to shell effects. That is, while the LEE contribution for the semi-magic nucleus ${ }^{51} \mathrm{~V}$ slightly increases, there is relatively a much larger enhancement of $\sigma_{-2}$ values for ${ }^{45} \mathrm{Sc}$ and ${ }^{56} \mathrm{Fe}$.

Conclusively, $\sigma_{-2}$ values can be assigned as a new spectroscopic probe to extract information on shell effects at high-excitation energies; in the same way as atomic masses are useful to study nuclear structure via nuclear binding energies. Finally, the observed deviations from the actual GDR effects may support the validity of 1) recent large-scale SM calculations, which predict the $M 1$ nature of the LEE, and 2) the generalized Brink-Axel hypothesis, which surprisingly, also allows for structural changes. This work emphasizes the need for new photo-absorption cross-section and $f\left(E_{\gamma}\right)$ measurements, and opens a new research avenue to investigate the existence and evolution of magic numbers at high-excitation energies from $\sigma_{-2}$ values [41].

Acknowledgements This work was supported by the National Research Foundation of South Africa under Grant 93500, the MaNus/MatSci program and the SA-CERN collaboration.

Open Access This article is distributed under the terms of the Creative Commons Attribution 4.0 International License (http://creativecommons.org/licenses/by/4.0/), which permits unrestricted use, distribution, and reproduction in any medium, provided you give appropriate credit to the original author(s) and the source, provide a link to the Creative Commons license, and indicate if changes were made.

\section{References}

1. Migdal, A.: Quadrupole and dipole gamma-radiation of nuclei. J. Exptl. Theoret. Phys. U.S.S.R. 15, 81 (1945)

2. Steinwedel, H., Jensen, J.H.D., Jensen, P.: Nuclear dipole vibrations. Phys. Rev. 79, 1019 (1950)

3. Bethe, H.A., Bacher, R.F.: Stationary states of nuclei. Rev. Mod. Phys. 8, 82 (1936)

4. von Weizsäcker, C.F.: Zur Theorie der Kernmassen. Z. Phys. 96, 431 (1935)

5. Migdal, A.B., Lushnikov, A.A., Zaretsky, D.F.: Theory of dipole photoabsorption of nuclei. Nucl. Phys. A 66, $193(1965)$ 
6. Dietrich, S.S., Berman, B.L.: Atlas of photoneutron cross sections obtained with monoenergetic photons. At. Data Nucl. Data Tables 38, 199 (1988)

7. Orce, J.N.: New formulas for the $(-2)$ moment of the photoabsorption cross section, $\sigma_{-2}$, Phys. Rev. C 91, $064602(2015)$

8. Levinger, J.S.: Migdal's and Khokhlov's calculations of the nuclear photoeffect. Phys. Rev. 107, 554 (1957)

9. Gaardhøje, J.J.: Nuclear structure at high excitation energy studied with giant resonances. Annu. Rev. Nucl. Part. Sci. 42, 483 (1992)

10. Eichler, J.: Second-order coulomb excitation via the giant dipole resonance. Phys. Rev. 133, B1162 (1964)

11. de Boer, J., Eichler, J.: The reorientation effect. Adv. Nucl. Phys. 1, 1 (1968)

12. Häusser, O.: Nuclear Spectroscopy and Reactions C, edited by J. Cerny. Academic, New York (1974)

13. Alder, K., Winther, A.: Electromagnetic Excitation. North-Holland, Amsterdam (1975)

14. Häusser, O. et al.: E1 polarization in coulomb excitation of ${ }^{7} \mathrm{Li}$. Nucl. Phys. A 212, 613 (1973)

15. Orce, J.N., et al.: Reorientation-effect measurement of the $\left\langle 2_{1}^{+}\|\mathrm{E} 2\| 2_{1}^{+}\right\rangle$matrix element in ${ }^{10}$ Be. Phys. Rev. C 86, 041303(R) (2012)

16. Raju, M.K. et al.: Reorientation-effect measurement of the first $2^{+}$state in ${ }^{12} \mathrm{C}$ : confirmation of oblate deformation. Phys. Lett. B. 777, 250 (2018)

17. Knüpfer, W., Richter, A.: Effect of the nuclear magnetic susceptibility on the evaluation of the electric dipole polarizability. Phys. Lett. B 107, 325 (1981)

18. Knüpfer, W., Richter, A.: On the scaling of the paramagnetic susceptibility of atoms, nuclei and nucleons. Z. Phys. A 320, 253 (1985)

19. Ahrens, J., Gimm, H., Zieger, A., Ziegler, B.: Experimental values for the static nuclear polarizability. Il Nuovo Cimento A 32(3), 364 (1976)

20. Voinov, A., et al.: Large enhancement of radiative strength for soft transitions in the quasicontinuum. Phys. Rev. Lett. 93, 142504 (2004)

21. Schiller, A. et al.: Extraction of level density and $\gamma$ strength function from primary $\gamma$ spectra. Nucl. Instr. Meth. Phys. Res. A 447, 498 (2000)

22. Guttormsen, M., et al.: Radiative strength functions in ${ }^{93-98}$ Mo. Phys. Rev. C 71, 044307 (2005)

23. Wiedeking, M., et al.: Low-energy enhancement in the photon strength of ${ }^{95}$ Mo. Phys. Rev. Lett. 108, 162503 (2012)

24. Brink, D.: Doctoral Thesis. Oxford University (1955)

25. Axel, P.: Electric dipole ground-state transition width strength function and 7-MeV photon interactions. Phys. Rev. 126, 671 (1962)

26. Jones, M.D., et al.: Examination of the low-energy enhancement of the $\gamma$-ray strength function of ${ }^{56} \mathrm{Fe}$. Phys. Rev. C 97, 024327 (2018)

27. Schwengner, R., Frauendorf, S., Larsen, A.C.: Low-energy enhancement of magnetic dipole radiation. Phys. Rev. Lett. 111, 232504 (2013)

28. Brown, B.A., Larsen, A.C.: Large Low-Energy M1 Strength for ${ }^{56,57}$ Fe within the Nuclear Shell Model. Phys. Rev. Lett. 113, 252502 (2014)

29. Sieja, K.: Electric and magnetic dipole strength at low energy. Phys. Rev. Lett. 119, 052502 (2017)

30. Sieja, K.: Low energy dipole strength from large scale shell model calculations. EPJ Web of Conferences 146, 05004 (2017)

31. Midtbo, J.E., et al.: Consolidating the concept of low-energy magnetic dipole decay radiation. Phys. Rev. C 98, 064321 (2018)

32. Larsen, A.C., et al.: Enhanced low-energy $\gamma$-decay strength of ${ }^{70} \mathrm{Ni}$ and its robustness within the shell model. Phys. Rev. C 97, 054329 (2018)

33. Litvinova, E., Belov, N.: Low-energy limit of the radiative dipole strength in nuclei. Phys. Rev. C 88, 031302(R) (2013)

34. Jackson, J.D.: Classical Electrodynamics. Wiley, Hoboken (1962)

35. Larsen, A.C., et al.: Transitional $\gamma$ strength in Cd isotopes. Phys. Rev. C 87, 014319 (2013)

36. Kheswa, B.V. et al.: Galactic production of ${ }^{138} \mathrm{La}$ : Impact of ${ }^{138,139}$ La statistical properties. Phys. Lett. B 744, 268 (2015)

37. Simon, A., et al.: First observation of low-energy $\gamma$-ray enhancement in the rare-earth region. Phys. Rev. C 93, 034303 (2016)

38. Bartholomew, G.A., Earle, E.D., Ferguson, A.J., Knowles, J.W., Lone, M.A.: Gamma-ray strength functions. Adv. Nucl. Phys. 7, 229 (2012)

39. Guttormsen, M., et al.: Validity of the generalised Brink-Axel hypothesis. Phys. Rev. Lett. 116, 012502 (2016) 
40. Schiller, A., Thoennessen, M.: Compilation of electric dipole resonances built on excited states. Atom. Data Nucl. Data Tables 93, 549 (2007)

41. Ngwetsheni, C., Orce, J.N.: Continuing influence of shell effects at high-excitation energies. Phys. Lett. B 792, 335 (2019)

42. Vessiere, A. et al.: A study of the photoneutron contribution to the giant dipole resonance of sd shell nuclei. Nucl. Phys. A 227, 513 (1974)

43. Larsen, A.C., et al.: Nuclear level densities and $\gamma$-ray strength functions in ${ }^{44,45}$ Sc. Phys. Rev. C 76, $044303(2007)$

44. Shoda, K.: $(\gamma, \mathrm{p})$ cross sections and isospin splitting of the giant dipole resonance in $\mathrm{N}=50$ nuclei. Nucl. Phys. A 239, 397 (1975)

45. Fultz, S.C. et al.: Photoneutron cross sections for ${ }^{51} \mathrm{~V}$ and ${ }^{59} \mathrm{Co}$. Phys. Rev 128, 2345 (1962)

46. Larsen, A.C., et al.: Microcanonical entropies and radiative strength functions of ${ }^{50,51}$ V. Phys. Rev. C 73, 064301 (2006)

47. Borodina, S.S. et al.: ${ }^{54,56} \mathrm{Fe}$ and ${ }^{58,60} \mathrm{Ni}(\gamma, \mathrm{n}),(\gamma, \mathrm{p}),(\gamma, \mathrm{np})$, and $(\gamma, 2 \mathrm{n})$ reaction cross sections evaluation using the model of the GDR state decay channel competition phenomenological description. Moscow State Univ. Inst. of Nucl. Phys. Rep., 6 (2000)

48. Larsen A.C., et al.: Evidence for the dipole nature of the low-energy $\gamma$ Enhancement in ${ }^{56} \mathrm{Fe}$. Phys. Rev. Lett. 111, 242504 (2013)

49. Carlos, P. et al.: A study of the photoneutron contribution to the giant dipole resonance of nuclei in the $64 \leqq \mathrm{~A} \leqq 86 \mathrm{~m}$ ass region. Nucl. Phys. A 258, 365 (1976)

50. Spyrou, A., et al.: Novel technique for constraining r-process $(\mathrm{n}, \gamma)$ reaction rates. Phys. Rev. Lett. 113, 232502 (2014)

51. Berman, B.L. et al.: Photoneutron cross sections for ${ }^{90} \mathrm{Zr},{ }^{91} \mathrm{Zr},{ }^{92} \mathrm{Zr},{ }^{94} \mathrm{Zr}$ and ${ }^{89} \mathrm{Y}$. Phys. Rev. 162, 1098 (1967)

52. Guttormsen, M., et al.: Quasicontinuum $\gamma$ decay of ${ }^{91,92} \mathrm{Zr}$ : benchmarking indirect $(\mathrm{n}, \gamma)$ cross section measurements for the s process. Phys. Rev. C 96, 024313 (2017)

53. Utsunomiya, H., et al.: M1 $\gamma$-strength for zirconium nuclei in the photoneutron channel. Phys. Rev. Lett. 100, 162502 (2008)

54. Beil, H. et al.: A study of the photoneutron contribution to the giant dipole resonance in doubly even Mo isotopes. Nucl. Phys. A 227, 427 (1974)

55. Utsunomiya, H., et al.: Photoneutron cross sections for Mo isotopes: a step toward a unified understanding of $(\gamma, \mathrm{n})$ and $(\mathrm{n}, \gamma)$ reactions. Phys. Rev. C 88, 015805 (2013)

56. Beil, H. et al.: Giant dipole resonance in $\mathrm{N}=82$ nuclei. Nucl. Phys. A 172, 426 (1971)

57. Carlos, P. et al.: The giant dipole resonance in the transition region of the samarium isotopes. Nucl. Phys. A 225, 171 (1974)

58. Arnould, M., Goriely, S., Takahashi, K.: The r-process of stellar nucleosynthesis: astrophysics and nuclear physics achievements and mysteries. Phys. Rep. 450, 97 (2007)

59. Larsen, A.C., Goriely, S.: Impact of a low-energy enhancement in the $\gamma$-ray strength function on the neutron-capture cross section. Phys. Rev. C 82, 014318 (2010)

60. Larsen, A.C., Spyrou, A., Liddick, S.N., Guttormsen, M.: Novel techniques for constraining neutroncapture rates relevant for $r$-Process heavy-element nucleosynthesis. Prog. Part. Nucl. Phys 107, 69 (2019)

Publisher's note Springer Nature remains neutral with regard to jurisdictional claims in published maps and institutional affiliations. 